\title{
Etude de la capacité cellulolytique de l'escargot Helix aspersa nourri avec des rations semisynthétiques
}

\author{
J. FONOLLA et $M$. Remedios SANZ \\ avec la collaboration technique de Francisca GIL \\ Departamento de Fisiología animal \\ Estación experimental del Zaidín (C.S.I.C.) \\ Prof. Albareda, I Granada, España
}

\section{Résumé}

Les résultats concernant la détermination et l'évaluation de la capacité cellulolytique de l'escargot Helix aspersa, une des espèces les plus intéressantes pour l'élevage, sont présentés dans ce mémoire.

Les essais ont été effectués en conditions thermo-hygrométriques contrôlées. Les escargots ont consommé 4 rations contenant différentes quantités de cellulose microgranulaire $(0,5,10$ et $20 \mathrm{p} .100)$. On a déterminé l'ingestion d'aliment, les digestibilités de la matière sèche, de la fibre neutre-détergente (FND) et protéine, ainsi que le bilan azoté.

A la suite de l'étude, il semble que cet escargot soit capable de digérer jusqu'à $0,90 \mathrm{mg} \mathrm{FND} / \mathrm{g}$ poids vif/animal/jour.

Mots clés : Escargot, Helix, capacité cellulolytique, digestibilité, bilan azoté.

\section{Introduction}

Florkin \& Lozet (1949) rapportent que Bioderman en 1898 découvrit la présence de cellulase dans l'intestin des invertébrés, chez les escargots du genre Helix. Depuis lors, on a spéculé sur l'origine de la capacité cellulolytique des escargots (Florkin \& Lozet, 1949 ; Grove \& Newell, 1960 ; Stone \& Morton, 1958) jusqu'à ce que Parnas en 1961, démontra que l'origine est double en observant l'activité cellulolytique dans les glandes salivaires et l'estomac et aussi chez la glande digestive. Avec les antibiotiques, cette activité disparaît dans les glandes salivaires et l'estomac, mais non dans la glande digestive. Cette étude, ainsi que d'autres réalisées au cours des années suivantes (Tiunova et al., 1970; Marshall, 1973; Calow \& Calow, 1975 ; Marshall \& Grand, 1976 ; Dean, 1978 ; RaO, 1979), ont déterminé les caractéristiques physiques et chimiques de cette cellulase.

C'est en considérant l'élevage possible des escargots, surtout de certaines espèces, que l'étude de cette capacité enzymatique acquiert une grande importance. LAzARIDOUDimitriadou \& Daguzan (1978) affirment que l'assimilation élevée de l'aliment chez l'escargot Euparypha pisana peut être due à la présence d'enzymes qui hydrolysent les 
polysaccharides structurales des plantes, en délivrant de nouvelles sources d'énergie pour l'animal.

Eu égard à l'intérêt de ces études, nous avons effectué une série d'essais afin de déterminer et d'évaluer, si possible, la capacité cellulolytique d'une des espèces d'escargots les plus intéressantes pour l'élevage (Chevallier, 1980 ; Rousselet, 1977). A ce propos, nous avons préparé des rations semisynthétiques et effectué une série d'essais dans lesquels on a déterminé l'ingestion d'aliment, la digestibilité de la matière sèche et de la fibre neutre-détergente (FND) comme un index de l'utilisation digestive de la cellulose, parce que la FND comprend les composants de la paroi cellulaire des plantes parmi lesquels se trouve la cellulose (VAN SoEST \& WINE, 1967). Nous avons déterminé aussi la digestibilité et la rétention totale de la protéine moyennant le bilan d'azote, puisque l'utilisation de la protéine ingérée est indépendante de l'énergie disponible.

\section{Matériel et méthodes}

Les escargots ont été achetés au marché au début du printemps et l'espèce Helix aspersa a été sélectionnéc. Les animaux ont été distribués au hasard dans 8 groupes de 15. Chaque groupe a été placé à l'intérieur d'un châssis d'élevage en plastique vert, avec un couvercle garni d'un grillage à maille carrée afin que l'intérieur soit bien aéré et éclairé. La surface du châssis d'élevage était de $1 / 4 \mathrm{~m}^{2}$. Les essais ont ćté effectués dans une salle de climatisation et en conditions expérimentales contrôlées pour induire la croissance optimale (Chevallier, 1981) : température d'élevage $18 \pm 2{ }^{\circ} \mathrm{C}$; humidité relative de l'air, $96 \pm 2$ p. 100 ; photopériode, 12 heures d'éclairement artificiel.

Les animaux ont consommé 4 rations comportant différentes quantités de cellulose microgranulaire $(0,5,10$ et $20 \mathrm{p}$. 100) (tabl. 1). On a réalisé 5 expériences dans lesquelles chacun des 2 lots consomment au hasard seulement 1 des 4 rations. Les rations ont été administrées «ad libitum», dans des petites mangeoires de $2 \mathrm{~cm}$ de diamètre et $0,5 \mathrm{~cm}$ de hauteur. Les abreuvoirs, similaires aux mangeoires mais plus profonds, contenaient de l'eau et une goutte $(5 \mathrm{ml})$ de «Biominol A + D» (complćment hydrosoluble de vitamines $\mathrm{A}+\mathrm{D})$.

Le modèle expérimental est de type factoriel $4 \times 5$, dans lequel le premier facteur est la ration utilisée (le niveau de FND) et le deuxième, le temps de réalisation de chaque expérience. Par analyse de la variance, on a testé les effets principaux et les interactions entre les facteurs (SoKAL \& RoHLF, 1979). A la suite du traitement statistique des données, les valeurs moyennes ont été comparées avec un test « $t$ »de Tukey.

Chaque expérience a eu une durée totale de 14 jours, au cours de laquelle, on a contrôlé l'aliment. Pendant les 8 derniers jours on a récolté quotidiennement les fèces et le dernier jour l'urine et le mucus, moyennant lavage du châssis d'élevage avec de l'eau distillée. Les escargots ont été pesés au début et à la fin de chaque expérience. Les poids des animaux et la distribution des rations par lot et expérience figurent au tableau 2 .

On a déterminé dans les rations et les fèces : l'humidité, par perte de poids dans une étuve à $100 \pm 2{ }^{\circ} \mathrm{C}$ jusqu'à poids constant, l'azote par la méthode de Kjeldahl avec conversion en protéine moyennant le facteur 6,25 et la FND d'après la méthode de Van Soest \& Wine (1967). On a mesuré aussi l'azote des eaux de lavage. 


\section{TABLEAU 1}

Composition centésimale des régimes expérimentaux et résultats d'analyse.

Composition of the diets (p. 100) and analytical results.

\begin{tabular}{|c|c|c|c|c|}
\hline Rations - Diets & I & II & III & IV \\
\hline Farine de soja - Soyabean meal & 60 & 60 & 60 & 60 \\
\hline Cellulose - Cellulose ......... & - & 5 & 10 & 20 \\
\hline Amidor - Starch ... & 20 & 15 & 10 & - \\
\hline $\mathrm{CO} \mathrm{Ca}$ & 20 & 20 & 20 & 20 \\
\hline Matière sèche (MS) - Dry matter (DM) & 92,58 & 92,33 & 92,73 & 93,81 \\
\hline Matière organique (MO) - Organic matter $(O M)$ & 77,85 & 77,90 & 77,82 & 77,61 \\
\hline Protéine (MS) - Protein (DM) & 29,65 & 29,73 & 29,60 & 29,26 \\
\hline Fibre neutre-détergente (MS) - N.D.F. (DM) & 9,53 & 14,15 & 18,80 & 28,29 \\
\hline Cendres (MS) - Ash (DM) $\ldots \ldots \ldots \ldots \ldots$ & 22,15 & 22,10 & 22,18 & 22,39 \\
\hline
\end{tabular}

TABLeau 2

Distribution des rations par lots et expérience.

Allotment of diets per group and experiment.

\begin{tabular}{|c|c|c|c|c|c|}
\hline $\begin{array}{l}\text { Expériences } \\
\text { Experiments }\end{array}$ & 1 & 2 & 3 & 4 & 5 \\
\hline Ration - Diet & Lot & Lot & Lot & Lot & Lot \\
\hline I & $3-7$ & $2-5$ & $4-6$ & $1-4$ & $3-5$ \\
\hline II & $1-2$ & $6-8$ & $1-7$ & $6-7$ & $2-7$ \\
\hline $111 \ldots \ldots \ldots \ldots$ & $5-6$ & $1-4$ & $3-5$ & $2-8$ & $6-8$ \\
\hline IV & $4-8$ & $3-7$ & $2-8$ & $3-5$ & $1-4$ \\
\hline
\end{tabular}

Poids final ( $g$ ) des lots.

Final weight $(\mathrm{g})$ of groups.

\begin{tabular}{|c|c|c|c|c|c|}
\hline & 1 & 2 & 3 & 4 & 5 \\
\hline \multicolumn{6}{|c|}{ Lots } \\
\hline 1 & 59,7 & 67,1 & 72,1 & 71,6 & 70,4 \\
\hline 2 & 59,0 & 64,9 & 67,5 & 66,6 & 66,2 \\
\hline 3 & 49,0 & 52,1 & 56,2 & 58,0 & 59,1 \\
\hline 4 & 62,7 & 66,2 & 71,2 & 68,7 & 67,3 \\
\hline 5 & 60,1 & 61,2 & 64,5 & 62,0 & 63,9 \\
\hline 6 & 57,6 & 60,0 & 63,5 & 63,2 & 63,4 \\
\hline 7 & 66,0 & 71,3 & 75,8 & 75,8 & 76,9 \\
\hline 8 & 74,3 & 79,1 & 82,2 & 81,8 & 83,2 \\
\hline
\end{tabular}




\section{Résultats}

Les tableaux 3, 4 et 5 ainsi que les figures 1,2 et 3 montrent les valeurs moyennes et les erreurs-standard correspondantes, pour les différentes rations et expériences, des coefficients de digestibilité de la matière sèche (MS), de la fibre neutredétergente (FND) et des protéines (tabl. 3, fig. 1) ; les résultats de bilan azoté (N retenu/ingéré, p. 100, ou $\mathrm{N}$ retenu/absorbé, p. 100) (tabl. 4, fig. 2) et l'ingestion d'aliment, la FND digérée et l'azote retenu en $\mathrm{mg} / \mathrm{g}$ poids vif/animal/jour apparaissent sur le tableau 5 et la figure 3 .
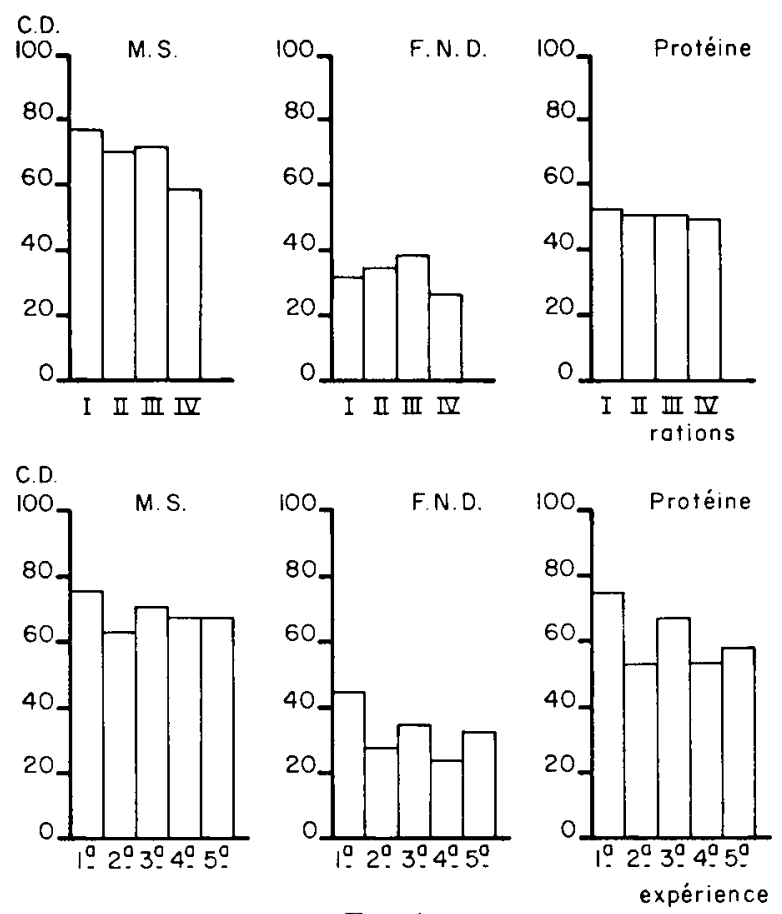

FIG. 1

Coefficients de digestibilité $(C D)$.

Digestibility coefficients (DC).

A la suite du traitement statistique on déduit que les facteurs ration et temps de réalisation de chaque expérience sont des effets indépendants. Seule, l'interaction dans le cas de $\mathrm{N}$ retenu (mg/g poids vif/animal/jour) est significative. 

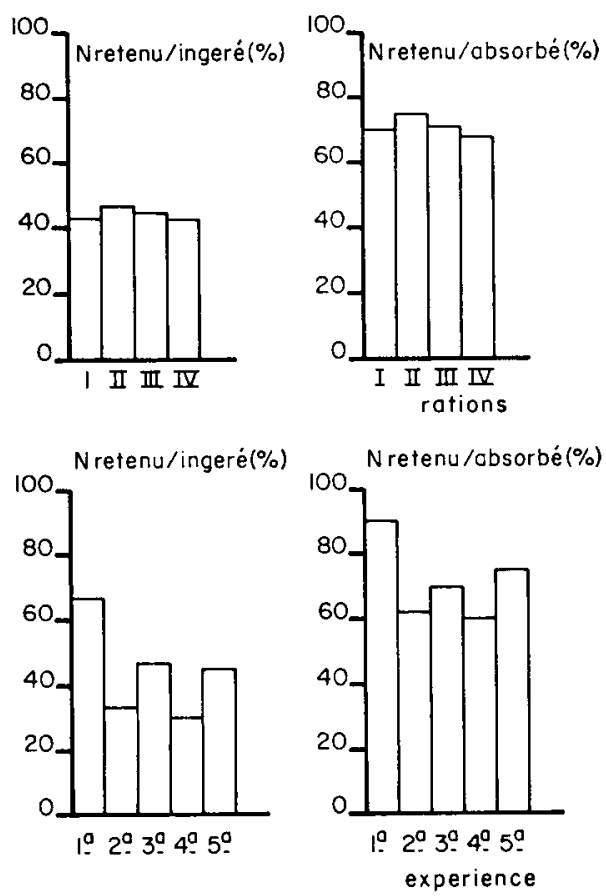

FIG. 2

Bilan azoté.

Nitrogen balance.
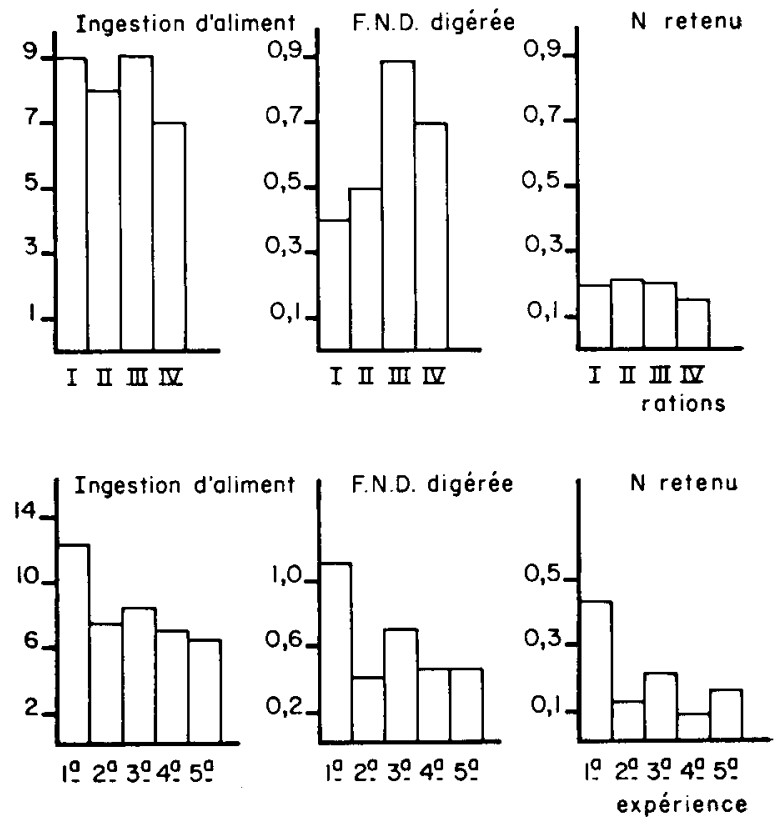

FIG. 3

Valeurs en $\mathrm{mg} / \mathrm{g}$ poids vif/animal/jour.

Values in $\mathrm{mg} / \mathrm{g}$ body weight/animal/day. 


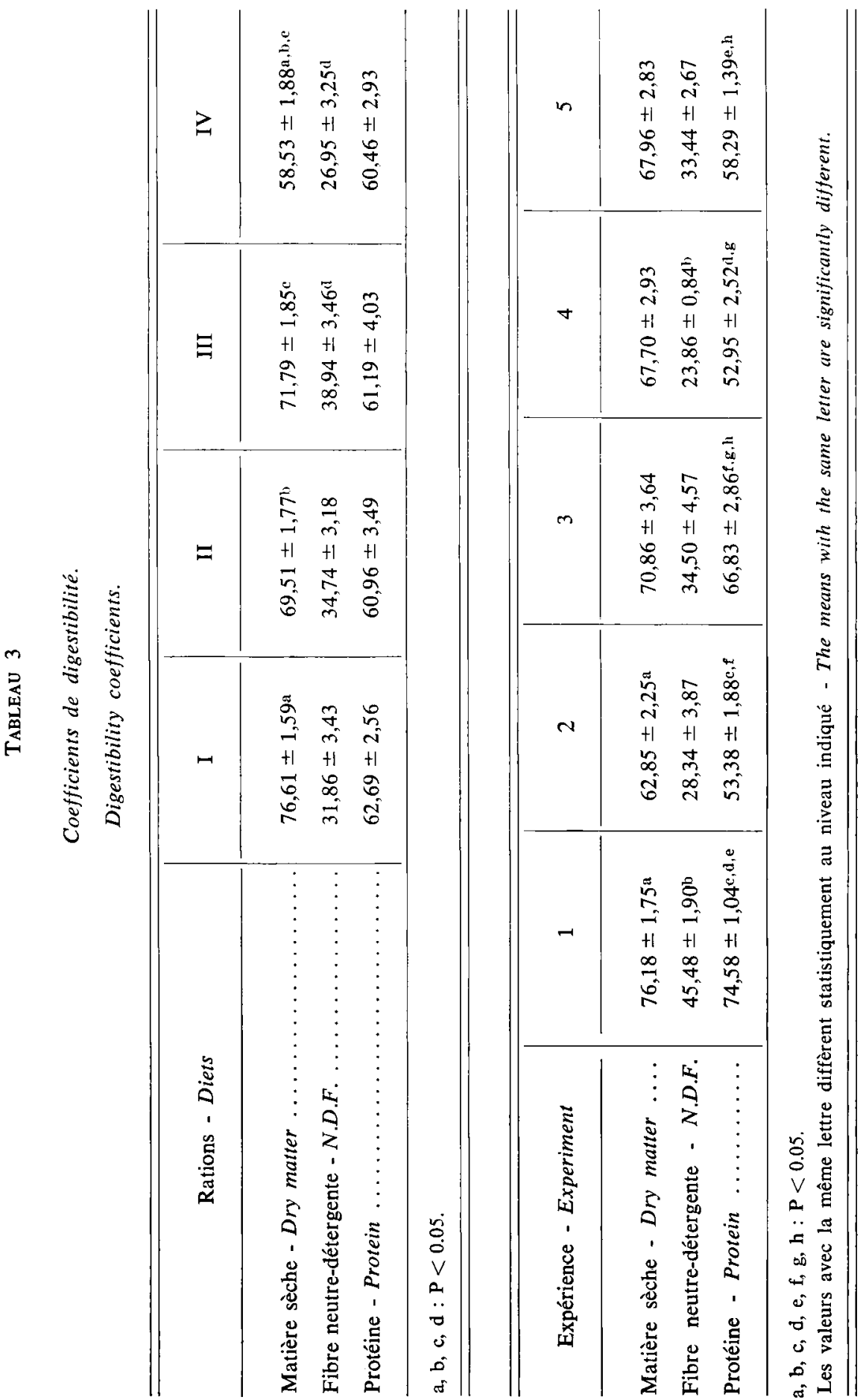




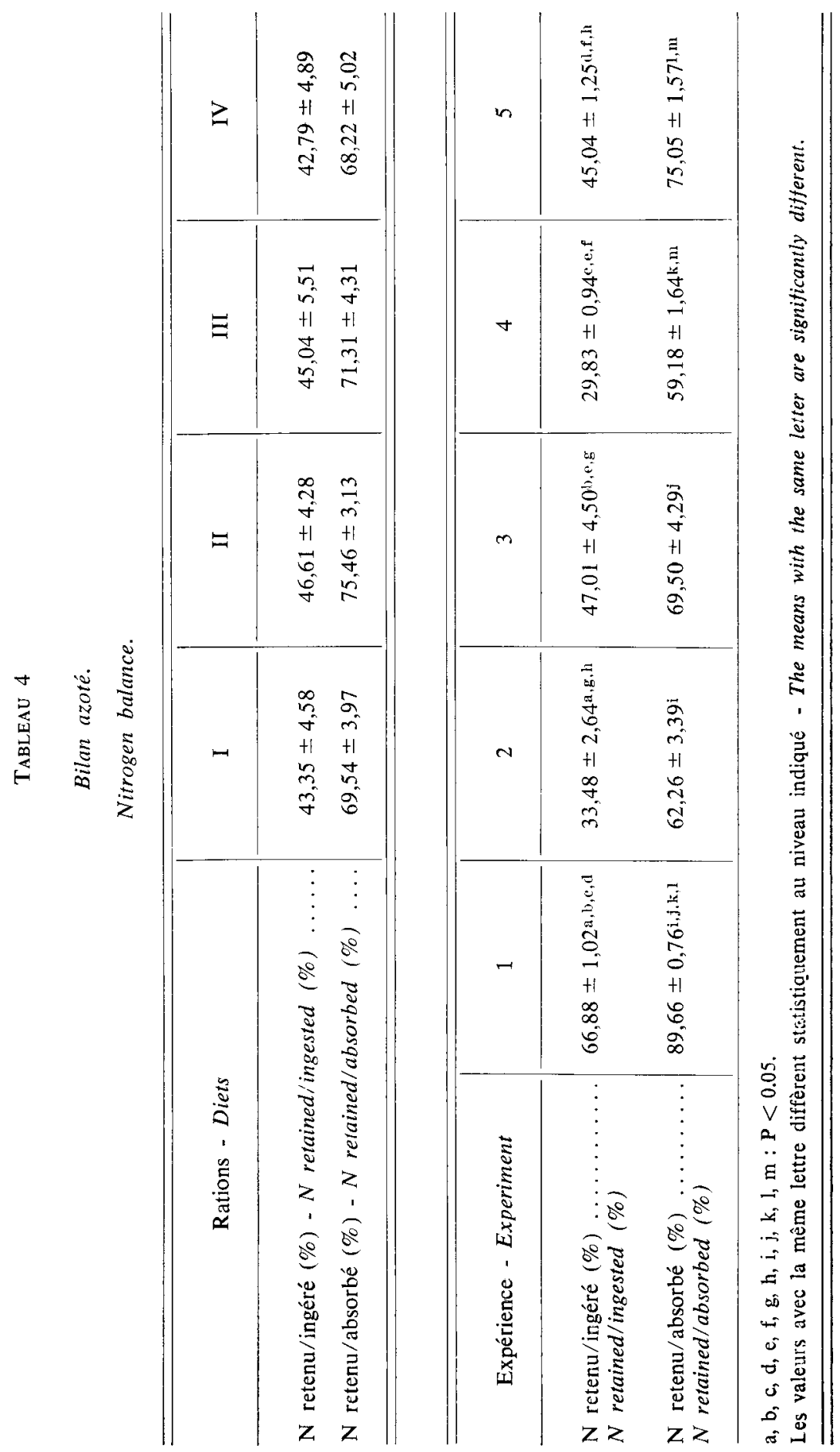




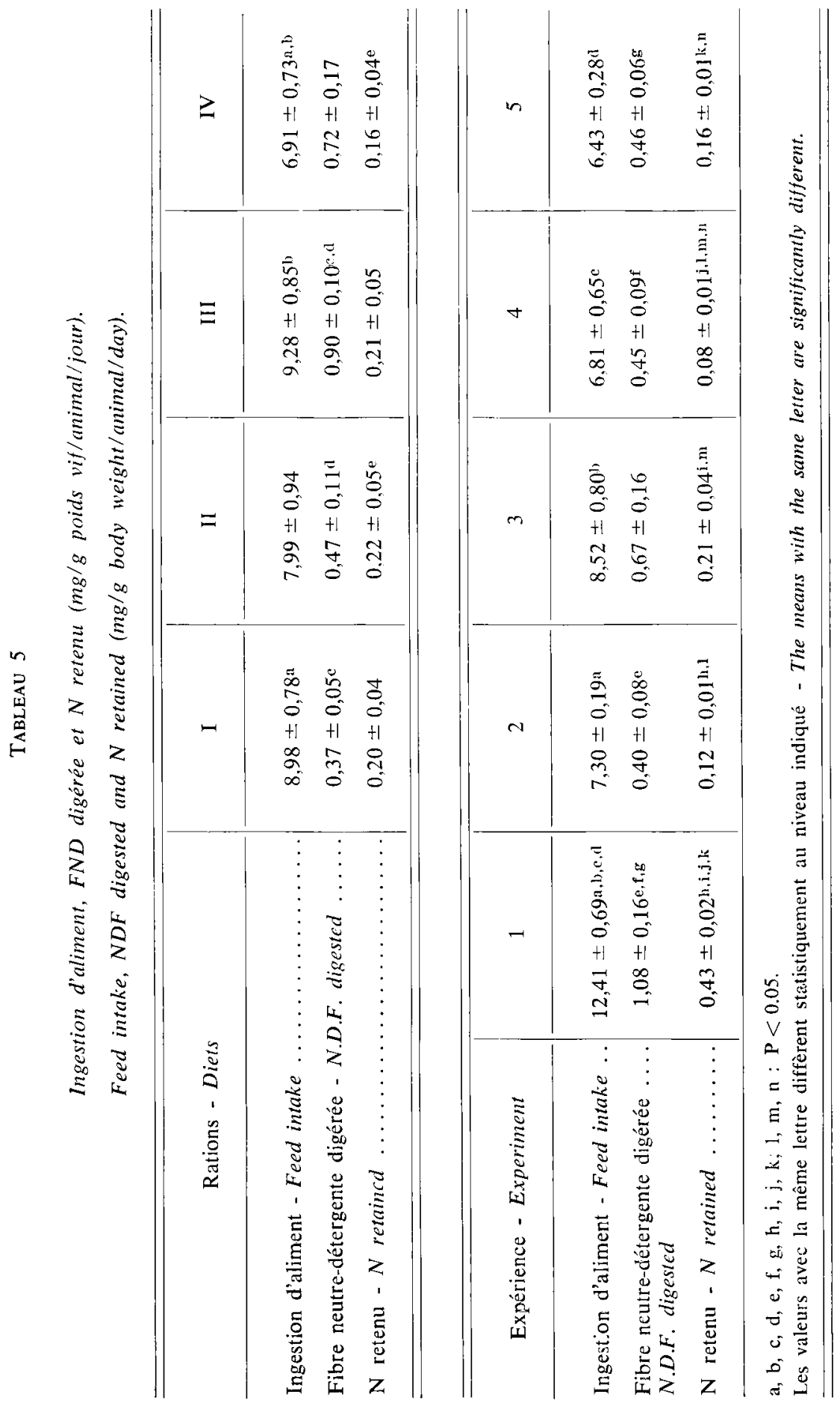




\section{Discussion et conclusions}

Les valeurs d'ingestion d'aliment sont en accord avec celles de Charrier \& DAgUZAN (1980) chez Helix aspersa en période de croissance et avec celles de FonOLLA et al. (1980) chez les escargots Helix aspersa adultes.

Le coefficient de digestibilité de la matière sèche, équivalent au rendement d'assimilation, est aussi très proche de ceux obtenus par certains auteurs chez Helix aspersa, et d'autres espèces, nourries d'aliments frais et déshydratés (STERN, 1969; Charrier \& Daguzan, 1980 ; Lazaridou-Dimitriadou \& Daguzan, 1978 ; Richardson, 1975 ; Scheerboom, Hemminga \& Doderer, 1978 ; Scheerboom \& Geldof, 1978), mais très supérieur, par contre, à ceux obtenus par les auteurs de cette étude dans une expérience antérieure (Fonolla et al., 1980), la différence pouvant être due à un défaut de matières minérales et de vitamines dans les rations utilisées dans l'expérience mentionnée.

Les résultats les plus significatifs correspondent à l'ingestion d'aliment et à la FND digérée en $\mathrm{mg} / \mathrm{g}$ poids vif/animal/jour (tabl. 5 et fig. 3), lesquelles sont très supérieures avec la ration III et inférieures avec la ration IV. Cet effet est significatif pour l'ingestion d'aliment mais non pour la FND digérée, ce qui pourrait être dû à la dispersion des données individuelles.

En ce qui concerne la digestibilité de la protéine et le bilan azoté ( $\mathrm{N}$ retenu/ingéré, p. 100 ou $\mathrm{N}$ retenu/absorbé, p. 100) (tabl. 3 et 4 et fig. 1 et 2), il n'y a pas de grandes différences entre les rations, mais quant à la rétention en $\mathrm{mg} / \mathrm{g}$ poids vif/animal/jour (tabl. 5 et fig. 3), la valeur correspondant à la ration IV est significativement inférieure à celle de la ration II, qui généralement est la plus haute, sauf dans la troisième expérience pour laquelle c'était la ration III, produisant done une interaction significative. Nous pensons que de plus grandes différences entre les rations pourraient se manifester en cas d'utilisation des rations avec des quantités moindres de protéines (celles des expériences contenaient environ 30 p. 100 de protéines).

Par conséquent, il apparaît que, dans les conditions de nos expériences, l'Helix aspersa est capable de digérer jusqu'à $0,90 \mathrm{mg}$ FND/g poids vif/animal/jour (ration III). C'est pour la ration IV que nous avons eu les plus faibles ingestions, FND digérée et rétention d'azote. Il aurait été souhaitable, à titre comparatif et pour compléter l'évaluation de la capacité cellulolytique d'Helix aspersa d'avoir utilisé une ration avec une composition en matières cellulosiques intermédiaire entre les rations III et IV.

C'est dans la première expérience qu'on observe la plus grande ingestion d'aliment, ceci avec toutes les rations; ces données concordent avec celles de ScheErboom, HemMINGA \& Doderer (1978), qui ont obtenu chez Lymnaea stagnalis une ingestion d'aliment très grande lorsqu'une ration à base de laitue était remplacée par une autre comprenant une grande quantité d'hydrates de carbone. Au début, les escargots ont ingéré la même quantité d'aliment, mais ensuite, du fait de la nature du nouveau régime, ils réduisaient peu à peu leur ingestion. D'après Scheerboom, Hemminga \& Doderer (1978) et Scheerboom \& Geldof (1978), l'ingestion d'aliments est réglée 
chez Lymnaea au moyen de deux mécanismes : d'abord une régulation par la stimulation des récepteurs mécaniques situés dans les glandes digestives et, plus tard, une autre selon la concentration de glucose dans l'hémolymphe. La régulation de l'ingestion dépend par conséquent du temps d'action des deux mécanismes.

\section{Summary}

The cellulolytic activity in the snail Helix aspersa fed with semi-synthetic diets

The aim of the present study was to determine the cellulolytic activity in the snail Helix aspersa, one of the most interesting specics for breeding purposes. Snails were kept in standard climatic conditions and fed with diets including different amounts of cellulose (table 1). The feed intake, digestibilities of dry matter, neutral detergent fibre (NDF) and protein as well as the nitrogen balance, were determined (tables 3,4 and 5 ; fig. 1, 2 and 3). It was concluded that the snail Helix aspersa is capable of digesting up to $0.90 \mathrm{mg} \mathrm{NDF} / \mathrm{g}$ live weight/animal/day.

Key words : Snail, Helix, cellulolytic activity, digestibility, nitrogen balance.

Reçu en mai 1983.

Accepté en novembre 1983.

\section{Références bibliographiques}

Calow P., Calow L.J., 1975. Cellulase activity and niche separation in fresh-water gastropods. Nature, 255, 478-480.

Charrier M., Daguzan J., 1980. Etude de la consommation alimentaire et de la production de l'escargot «Petit gris » Helix aspersa Müller (Gastéropode pulmoné terrestre) élevé sous abri. Haliotis, 10, 41-44.

Chevallier H., 1980. Les escargots du genre Helix commercialisés en France. Haliotis, 10, 11-23.

Chevallier H., 1981. Facteurs de croissance pour l'escargot Petit-Gris en élevage. L'Esc. Ecol., 13, 9-14.

DEAN R.C., 1978. Mechanisms of wood digestion in the Shipworm Bankia Gouldi Bartsch : Enzyme degradation of celluloses, hemicelluloses and wood cell walls. Biol. Bull., $155,297-316$.

Florkin M., Loze't F., 1949. Origine bactérienne de la cellulase du contenu intestinal de l'escargot. Arch. Int. Physiol., 57, 201-207.

Fonolla J., Sanz R., Prieto C., Guerrero J.E., 1980. Détermination de la valeur nutritive de différentes rations chez l'escargot Helix aspersa, dans des conditions écologiques contrôlées. Haliotis, 10, 37-40.

Grove A.J., Newell G.E., 1960. In : Animal Biology. 7th ed. Univ. Tutorial. Press Ltd. Clifton House, Euston Road, 864 p., London.

Lazaridou-Dimitriadou M., Daguzan J., 1978. Consommation alimentaire. Production et bilan énergétique chez Euparypha pisana (Müller) (Gastéropode pulmoné). Ann. Nutr. Alim., 32, 1317-1350.

Marshall J.J., 1973. Comparative studies on B-glucanhydrolases. I. Purification of a B-1-4-glucanhydrolase (cellulase) from the snail Helix pomatia. Comp. Biochem. Physiol. B., 44, 981-988. 
Marshall J.J., Grand R.J.A., 1976. Characterization of a B-1-4-glucanhydrolase from the snail, Helix pomatia. Comp. Biochem. Physiol. B., 53, 231-237.

Parnas I., 1961. The cellulolytic activity in the Snail Levantina hierosolyma Boiss. J. Cell. Comp. Physiol., 58, 195-201.

RaO M.B., 1979. Preliminary studies on the natural diet and carbohydrases in the digestive gland of the tropical aquatic pulmonate snail Limnaea luteola. Malacologia, 18, 421-422.

Richardson A.M.M., 1975. Food, Feeding rates and assimilation in the land snail Cepea nemoralis. Oecologia, 19, 59-70.

Rousselet M., 1977. L'escargot et l'héliciculture. Thèse. Paris. Univ. Val-de-Marne, 159 p.

Scheerboom J.E.M., Hemminga M.A., Doderer A., 1978. The effects of a change of diet on consumption and assimilation and on the haemolymph-glucose concentration of the pond snail Lymnaea stagnalis (L.). Zoology, 81, 1-12.

Scheerboom J.E.M., Geldor A.A., 1978. A quantitative study of the assimilation of different diets in the pond snail Lymnaea stagnalis (L.), introducing a method to prevent coprophagy. Zoology, 81, 173-183.

SoKal R.A., Rohlf F.J., 1979. In : Biometria. $1^{\text {a }}$ ed. H. Blume Ediciones, 832 p., Madrid.

Stern G., 1969. Bilan énergétique de la limace Arion rufus (Mollusque Pulmoné) en période de croissance. C.R. Acad. Sci. Paris, 269, 1015-1018.

Stone B.A., Morton J.E., 1958. The distribution of cellulases and related enzymes in Mollusca. Proc. Malacol. Soc. London, 33, 127-141.

Tiunova N.A., Rodionova N.A., Feniksova R.V., Martinovitch L.I., 1970. Decomposition of cellulose substrates by cellulase preparations. Appl. Biochem. Microbiol., 6, 437-443.

VAN SOEST P.J., Wine R.H., 1967. Use of detergents in the analysis of fibrous feeds. IV. Determination of plant cellwalls constituents. J. Assoc. Off. Agric. Chem., 50, 50-56. 